\title{
Graft Maturation of Autologous Chondrocyte Implantation
}

\section{Magnetic Resonance Investigation With T2 Mapping}

\author{
Thomas R. Niethammer, ${ }^{*}$ MD, Elem Safi, ${ }^{*}$ MD, Andreas Ficklscherer, ${ }^{*}$ MD, Annie Horng, ${ }^{\dagger}$ MD, \\ Markus Feist, ${ }^{*} \mathrm{MD}$, Isa Feist-Pagenstert, ${ }^{*} \mathrm{MD}$, Volkmar Jansson, ${ }^{*} \mathrm{MD}$, \\ Matthias F. Pietschmann, ${ }^{*}$ MD, and Peter E. Müller, ${ }^{* \neq}$ MD \\ Investigation performed at the Department of Orthopaedics, \\ Ludwig Maximilians University Munich, Munich, Germany
}

\begin{abstract}
Background: Autologous chondrocyte implantation (ACl) using tissue-engineered cartilage is a successful therapy for fullthickness cartilage lesions in the knee joint. However, in vivo graft maturation is still unclear.

Purpose: The aim of this prospective study was to analyze graft maturation after $\mathrm{ACl}$ in the knee using objective T2 mapping in correlation with the clinical outcomes within a 3-year postoperative course.

Study Design: Case series; Level of evidence, 4.

Methods: A total of 13 patients with isolated cartilage defects of the knee were treated with Novocart 3D, a matrix-based ACI procedure in the knee joint. The patients had complete data from International Knee Documentation Committee (IKDC) scores and MRI examinations for 6 to 36 months postoperatively. All cartilage defects were arthroscopically classified as Outerbridge grades III and IV. The mean area of the cartilage defect was $5.6 \mathrm{~cm}^{2}$. Postoperative clinical and MRI examinations were conducted at $6,12,24$, and 36 months after surgery. The modified magnetic resonance observation of cartilage repair tissue (MOCART) score was used to evaluate the quality and integration of the Novocart 3D implants on MRI. The T2 relaxation time values of the $\mathrm{ACl}$ graft and healthy native cartilage areas were determined to assess graft maturation using T2 mapping.
\end{abstract}

Results: The T2 relaxation times of the ACl graft showed significant improvement, with decreasing values from 41.6 milliseconds at 6-month follow-up to 32.4 and 30.9 milliseconds after 24 and 36 months, respectively. These values were similar to the T2 relaxation times of the native surrounding cartilage. There was no correlation between the clinical outcomes (IKDC score) and T2 relaxation time values.

Conclusion: The T2 relaxation time in the repaired tissue showed similar values compared with normal hyaline cartilage. Graft maturation after $\mathrm{ACl}$ in the knee joint needs at least 1 year, with ongoing adjustment of the T2 relaxation time values compared with native surrounding cartilage. A correlation between increasing ACl graft maturation and clinical outcomes (IKDC score) could not be found with the data available.

Keywords: cartilage; autologous chondrocyte implantation; T2 relaxation time; ACI; MRI

Autologous chondrocyte implantation (ACI) has now been used for almost 20 years for the treatment of multilayer, circumscribed cartilage defects. Based on the Brittberg et $\mathrm{al}^{5}$ technique of classic ACI with a periosteal flap (p-ACI), a new generation of chondrocyte transplantation was created. Now, almost 20 years later, modified techniques have changed the safety and efficiency of this method for the treatment of large circumscribed cartilage defects in the knee joint. Matrix-based ACI (mb-ACI) is an advancement of the classic p-ACI technique in which the chondrocytes are seeded on an absorbable matrix. This

The American Journal of Sports Medicine, Vol. 42, No. 9 DOI: $10.1177 / 0363546514538756$

(C) 2014 The Author(s) method has the advantage of a stable and 3-dimensional arrangement of the chondrocytes in the cartilage defect and allows a simplification of surgical handling. Several clinical long-term studies have verified the benefit of the mb-ACI technique. ${ }^{2,3,10,14,20}$

Magnetic resonance imaging (MRI) is the method of choice for the evaluation of articular cartilage in the $\mathrm{knee}^{4}$ and has been shown to be sensitive to morphological alterations at cartilage repair sites and for comparison with a healthy area. ${ }^{6,8,12,13}$ T2 mapping is a new MRI method used to determine the collagen structure and water content. ${ }^{6,8,12,13,29}$ It has been useful in the characterization of cartilage and long-term tracking in several studies. ${ }^{6,13}$ The noninvasive $\mathrm{T} 2$ relaxation time measurement permits the determination of the cartilage structure, analyzing the water and collagen content. Therefore, registered harmonization of the T2 
relaxation time values of the graft and the healthy surrounding cartilage indicates increasing graft maturation.

The duration of ACI graft maturation has not been verified in studies yet. Clinical and radiological diversity in maturation has been described in detail elsewhere. ${ }^{11,24,25,30}$ These studies analyzed the maturation of mb-ACI with high-resolution MRI without analyzing the biochemical properties and cartilage structure with T2 mapping.

The aim of this study was to analyze in vivo graft maturation after ACI in the knee with objective T2 mapping of the ACI grafts within a 3-year postoperative course. We also analyzed the clinical relevance of ACI graft maturation using a correlation between the clinical outcomes evaluated with International Knee Documentation Committee (IKDC) scores and T2 relaxation times. Our hypothesis was that ACI leads to healthy and stable cartilage regeneration, with cartilage properties that are similar to the collagen structure and water content of the native surrounding cartilage.

\section{MATERIALS AND METHODS}

\section{Patients}

In this study, 13 patients with isolated cartilage defects of the knee were treated with the mb-ACI technique. These cases were treated between 2004 and 2007 and were included in a previous study. ${ }^{18,21}$ With institutional review board approval, these patients were examined clinically and radiologically within $6,12,24$, and 36 months postoperatively. The cartilage defects were arthroscopically classified as Outerbridge grades III and IV. The mean \pm SD area of the cartilage defects measured $5.6 \pm 1.3 \mathrm{~cm}^{2}$. Cartilage defect localizations were distributed as the following: $53.8 \%(\mathrm{n}=7)$ of the treated cartilage defects were on the medial femoral condyle (anterior-central), $15.4 \%(\mathrm{n}=2)$ were on the lateral femoral condyle (anterior-central), and $30.8 \%(\mathrm{n}=4)$ were retropatellar.

The inclusion criteria of the study were patients with local cartilage defects classified as Outerbridge grades III and IV, between 2 and $15 \mathrm{~cm}^{2}$ in size, and age between 16 and 50 years with intact menisci and ligaments. Patients also showed a mechanical axis below $5^{\circ}$. All patients had full clinical and MRI data values from 6 to 36 months. Patients with osteoarthritis of the knee, joint instability, arthritis, corresponding chondral defects, or more than 2 focal cartilage defects and patients with incomplete data from clinical $(\mathrm{n}=7)$ and MRI $(\mathrm{n}=21)$ examinations were excluded from the study.

All cartilage defects were treated with a biphasic collagenous scaffold (Novocart 3D, TETEC AG). As a first step, 2 or 3 osteochondral plugs were taken from the intercondylar notch. These plugs were placed in a sterile nutrient solution and were sent to the manufacturer. After a cultivation time of approximately 4 weeks, the ACI grafts were implanted. After a perioperative antibiotic prophylaxis, the knee joint was exposed by parapatellar arthrotomy. Cartilage defects were debrided with curettes and prepared with punches. After careful debridement with preparation of a stable cartilage rim, the cartilage defects were measured in size. Then, the chondrocyte-seeded scaffolds were cut and inserted into the cartilage defect. Absorbable sutures were used to fix the ACI graft in the defect. Fibrin glue was not used.

Postoperative rehabilitation was carried out as follows: in cases with femoral cartilage defects, rehabilitation began with a continuous passive motion device after 24 hours of bed rest and drain removal. Weightbearing was limited to $20 \mathrm{~kg}$ for 6 weeks with increasing flexion. Rehabilitation for patellar defects was conducted with the patient using a knee brace with limited flexion of $30^{\circ}$ for 2 to 3 weeks, which was gradually increased in the weeks thereafter. Full weightbearing was allowed with full extension after wound healing. Moderate physical activities were not allowed earlier than 3 months postoperatively.

\section{Clinical Scores}

The International Cartilage Repair Society (ICRS) Cartilage Injury Standard Evaluation Form 2000 was used as a basis for the assessment of subjective and objective clinical parameters, as well as the 2000 IKDC subjective knee evaluation form, and the visual analog scale (VAS) was used for the registration of pain at rest and on movement. The data were collected preoperatively and postoperatively (after 6, 12, 24, and 36 months), and the results were recorded in the standardized ICRS form. The IKDC and VAS used in this study are established and validated scores in the follow-up examination of cartilage regeneration procedures and have shown a high rate of reliability and reproduction in several studies. ${ }^{9,22}$

\section{T2 Relaxation Times}

A follow-up MRI examination was performed after 6, 12, 24, and 36 months using a 1.5-T scanner (Magnetom Avanto, Siemens Medical) according to a predetermined protocol as described in previous studies. ${ }^{21} \mathrm{~A}$ standard protocol including proton density-weighted sequences in 3 planes and a coronal T1-weighted sequence as well as either a coronal or axial (depending on the location of mb-ACI) T1-weighted fast low-angle shot (FLASH) sequence with selective water excitation and T2 multiecho sequence was performed (repetition time/echo time, 3000/13.2 ms; 8 echos; resolution, $0.6 \times 3 \mathrm{~mm}^{3}$ ). The 3 -dimensional FLASH sequence is a validated sequence for the measurement of knee cartilage

\footnotetext{
${ }^{\ddagger}$ Address correspondence to Prof. Dr. med. Peter E. Müller, Department of Orthopaedic Surgery, Physical Medicine and Rehabilitation, University Hospital of Munich, Campus Grosshadern, Marchioninistrasse 15, 81377 Munich, Germany (e-mail: peter.mueller@med.uni-muenchen.de).

${ }^{*}$ Department of Orthopaedic Surgery, Physical Medicine and Rehabilitation, University Hospital of Munich, Grosshadern Campus, Munich, Germany.

${ }^{\dagger}$ Institute of Clinical Radiology, Ludwig Maximilians University Munich, Campus Grosshadern, Munich, Germany.

T.R.N. and E.S. contributed equally to this article and share first authorship.

T.R.N., A.F., M.F.P., and P.E.M. are members of the working group "Tissue Regeneration" of the German Society for Orthopaedic and Trauma Surgery.

The authors declared that they have no conflicts of interest in the authorship and publication of this contribution.
} 

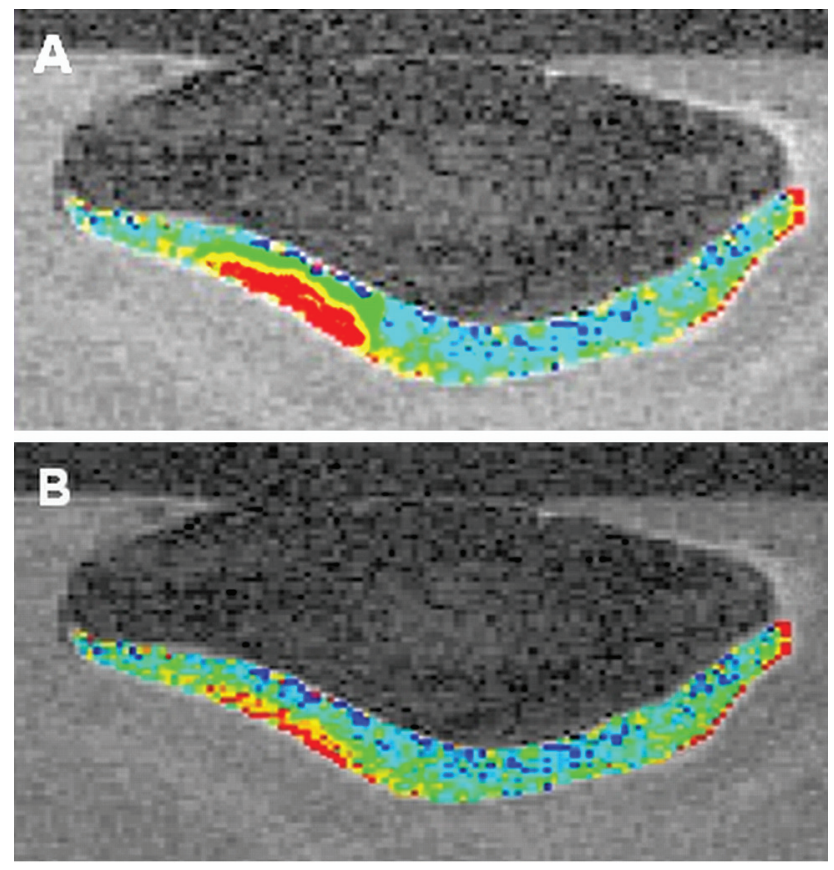

Figure 1. Magnetic resonance imaging in T2 patellar transversal view. Patellar cartilage is shown in colored T2 distribution (A) after 6 months and (B) after 12 months. Data sets were manually segmented and visualized in different sequences depending on the size of available tissue. The T2 relaxation time in patellar cartilage is visualized from 20 milliseconds (blue) to 50 milliseconds (red). The autologous chondrocyte implantation graft cartilage is well integrated into the surrounding native cartilage.

morphometry with MRI, as demonstrated by Eckstein et al. ${ }^{7}$ The measurements of cartilage were conducted in a blinded fashion by an experienced orthopaedic surgeon and a radiologist who specialized in musculoskeletal radiology.

The acquired Digital Imaging and Communications in Medicine (DICOM) data sets were transferred to a Unix workstation. Within the data sets, slices containing cartilage were manually segmented using the FLASH sequence. After the data were segmented, the cartilage plate was reconstructed 3-dimensionally through all segmented sequences. The next step contained the overlay of segmentation sequences with the multiecho data sets. Manual corrections were performed if differences between water excitation sequences and multiecho sequences were caused by movement of the patients during MRI examination. For T2 calculation, the segmented cartilage was subdivided into 3 zones from the subchondral bone to the cartilage surface and into 9 sections from the medial to the lateral border, resulting in 27 regions of interest (ROIs), with 70 picture elements per ROI for each cartilage plate (Figure 1).

\section{Statistical Analysis}

The retrieved data were analyzed using the statistics program SPSS (v 19, IBM Corp). The descriptive data are presented as the absolute and relative frequency of occurrence (mean $\pm \mathrm{SD}$ ). The coherence between clinical and radiological factors to the various follow-up examinations was verified with multiple logistic regression analysis. Between preoperative and postoperative examination times, significant differences were determined using the Wilcoxon test for dependent samples and the Mann-Whitney $U$ test for independent samples in the comparison of 2 groups at a certain time period. The level of significance was set at $P<.05$.

\section{RESULTS}

The mean age of the patients was $32.9 \pm 9.4$ years, and the mean body mass index was $23.7 \pm 3.4 \mathrm{~kg} / \mathrm{m}^{2}$ (range, 19.0 $30.1 \mathrm{~kg} / \mathrm{m}^{2}$ ). Cartilage defect localizations were distributed as follows: $9(69.2 \%)$ of the cartilage defects treated were femoral, and $4(30.8 \%)$ were retropatellar. Femoral cartilage damage occurred predominantly on the medial femoral condyle $(n=7)$, and the other 2 defects occurred on the lateral femoral condyle. The mean cartilage defect sizes were $5.6 \pm$ $1.3 \mathrm{~cm}^{2}$ (femoral) and $5.6 \pm 1.5 \mathrm{~cm}^{2}$ (retropatellar).

\section{T2 Relaxation Times}

In our 13 patients, we measured a mean $\mathrm{T} 2$ relaxation time of $30.9 \pm 3.6$ milliseconds for graft cartilage after 36 months (Table 1). Compared with values at 6-month follow-up, ACI graft cartilage showed significantly decreasing relaxation times after 24 months $(P=.000)$ and 36 months $(P=.002)$. Significant changes were also seen after 24 and 36 months compared with the values at 12-month follow-up ( $P=.03$ for both). No significant improvements were seen for ACI graft cartilage within 6- and 12-month follow-ups $(P=.2)$.

The T2 relaxation time values between ACI graft cartilage and surrounding native cartilage showed significant differences at 6 months postoperatively. From this point, no significantly different T2 relaxation time values between ACI graft cartilage and healthy surrounding cartilage could be found, with almost the same $\mathrm{T} 2$ relaxation time values (Figure 2). The $\mathrm{T} 2$ relaxation time ratio between ACI graft cartilage and native surrounding cartilage dropped from 1.23 after 6 months and continued to 0.94 after 36 months (Table 1 ).

\section{Clinical Evaluation}

The 13 patients from our study collectively showed a significantly higher IKDC score postoperatively after 6, 12, 24, and 36 months compared with the preoperative values (Figure 3). The mean preoperative IKDC score was $29.7 \pm 25.5$. During the postoperative observation period, the mean IKDC score increased to $57.3 \pm 17.0$, with the greatest significance at 24 and 36 months postoperatively. From 24 months postoperatively, the IKDC score showed stable results without significant changes.

During the postoperative follow-up examinations, the IKDC score and T2 relaxation time demonstrated no 
TABLE 1

T2 Relaxation Times for Healthy Cartilage and Graft Cartilage

\begin{tabular}{lcc}
\hline Follow-up & T2 Relaxation Time, Mean (Range), ms & $P$ Value \\
\hline 6 months & $33.7(25.9-40.6)$ & .01 \\
$\quad$ Healthy cartilage & $41.6(29.1-66.3)$ & 1.23 \\
$\quad$ Graft cartilage & $32.8(26.3-40.9)$ & .07 \\
12 months & $39.3(27.9-72.3)$ & 1.20 \\
Healthy cartilage & $34.1(26.6-53.1)$ & .19 \\
$\quad$ Graft cartilage & $32.4(23.4-58.1)$ & 0.95 \\
24 months & $32.9(27.2-38.9)$ & .25 \\
Healthy cartilage & $30.9(26.7-41.1)$ & 0.94 \\
Graft cartilage & & \\
Healthy cartilage & & \\
Graft cartilage & & \\
\hline
\end{tabular}

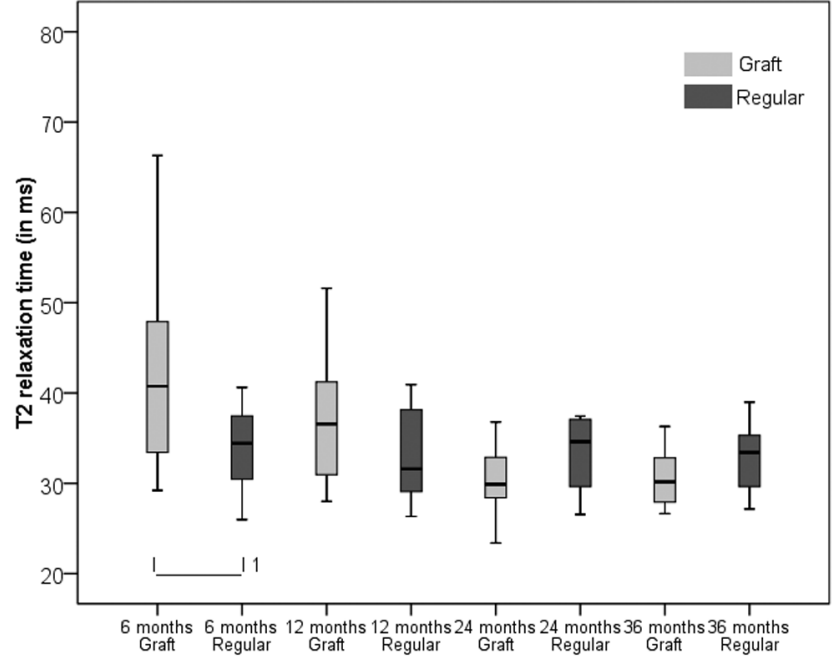

Figure 2. Overview of the T2 relaxation time values between autologous chondrocyte implantation (ACl) "graft" cartilage and "regular" surrounding native cartilage showed significant differences 6 months postoperatively. From this point, no significantly different $\mathrm{T} 2$ relaxation time values between $\mathrm{ACl}$ graft cartilage and healthy surrounding cartilage could be found, with almost the same T2 relaxation time values. Outliers are marked with circles.

significant correlation $(P=.70$ at 6 months; $P=.54$ at 12 months; $P=.66$ at 24 months; $P=.80$ at 36 months). The VAS score for pain at rest showed significantly lower results after $6,12,24$, and 36 months compared with the preoperative findings (3.4 preoperatively vs 1.3 after 36 months; $P=.03$ ) (Table 2). The VAS score for pain on movement also showed significant improvement, from 7.2 preoperatively to 4.7 after 6 months $(P=.02), 3.9$ after 12 months $(P=.001), 4.3$ after 24 months $(P=.01)$, and 4.7 after 36 months $(P=.05)$.

\section{DISCUSSION}

The most important finding of the present study was that T2 relaxation time values showed an increasing

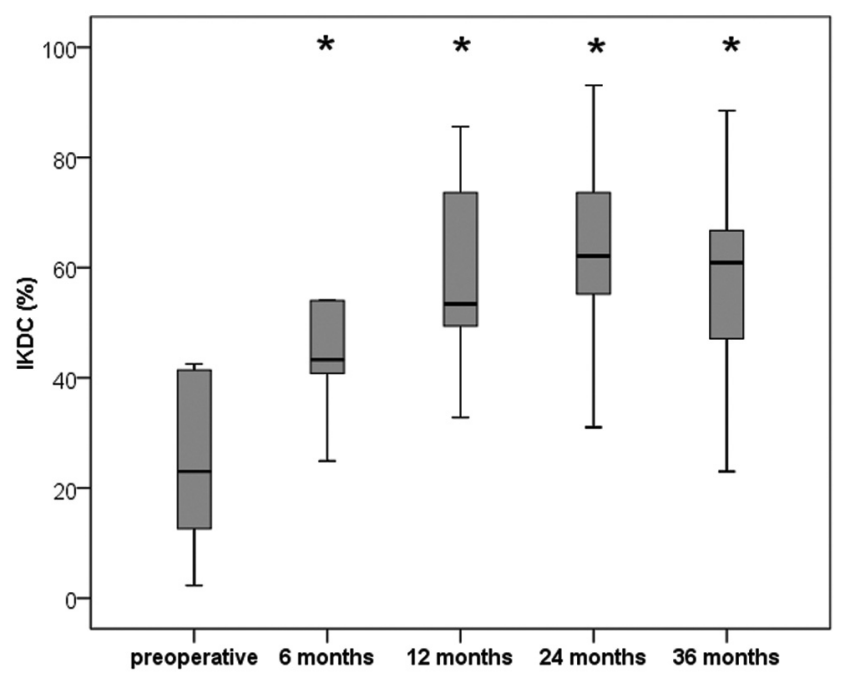

Figure 3. Overview of the clinical International Knee Documentation Committee (IKDC) scores, with significant improvement $(*)$ after $6(P=.04), 12(P=.006), 24(P=$ $.002)$, and $36(P=.005)$ months in comparison with the preoperative results (Wilcoxon test).

harmonization between ACI graft cartilage and the native surrounding cartilage, with significant improvement of the ACI graft values over the observation period of 3 years. The treatment of full-thickness cartilage defects still represents a challenge. Autologous chondrocyte implantation has provided a well-established method for the treatment of full cartilage defects for almost 2 decades. ${ }^{5,16}$ However, the maturation process of the ACI graft is still unclear. At present, little is known about the influence of ACI graft maturation and its clinical relevance. Noninvasive, conventional MRI is the method of choice for the evaluation of articular cartilage in the knee and has been shown to be sensitive to morphological alterations at cartilage repair sites. ${ }^{6,8,12,13}$

The measurement of $\mathrm{T} 2$ relaxation time is a suitable method to determine the structure of cartilage, analyzing the water and collagen content, and has been useful in the characterization of cartilage and long-term tracking 
TABLE 2

Overview of VAS Scores for Pain at Rest and on Movement ${ }^{a}$

\begin{tabular}{lccc}
\hline & & \multicolumn{2}{c}{ VAS Score for Pain ${ }^{b}$} \\
\cline { 3 - 4 } Follow-up & $\begin{array}{c}\text { IKDC Subjective } \\
\text { Score }\end{array}$ & $\begin{array}{c}\text { On Movement } \\
(\mathrm{n}=13)\end{array}$ & $\begin{array}{c}\text { At Rest } \\
(\mathrm{n}=13)\end{array}$ \\
\hline Preoperative & $29.7 \pm 25.5$ & $7.2 \pm 2.7$ & $3.4 \pm 2.9$ \\
6 months & $48.7 \pm 16.3$ & $4.7 \pm 2.7$ & $1.0 \pm 2.2$ \\
12 months & $58.1 \pm 16.0$ & $3.9 \pm 2.2$ & $1.2 \pm 1.9$ \\
24 months & $60.2 \pm 19.3$ & $4.3 \pm 2.6$ & $1.2 \pm 2.2$ \\
36 months & $57.3 \pm 17.0$ & $4.7 \pm 2.6$ & $1.3 \pm 1.7$ \\
\hline
\end{tabular}

${ }^{a}$ Values are expressed as mean $\pm \mathrm{SD}$. IKDC, International Knee Documentation Committee; VAS, visual analog scale.

${ }^{b}$ Significant improvements were seen after $6(P=.02), 12(P=$ $.001), 24(P=.01)$, and 36 months $(P=.05)$ compared with preoperative values for pain on movement. Also, significant improvements could be detected after $6(P=.03), 12(P=.03), 24(P=$ $.01)$, and 36 months $(P=.03)$ compared with preoperative values for pain at rest.

in several studies. ${ }^{1,11,25,29}$ Many different T2 relaxation times have been published in the literature. The most frequently measured time ranges are between 30 to 35 milliseconds for healthy cartilage and 34 to 41 milliseconds in knees with cartilage defects. ${ }^{6,19}$ For the first time, in this study, we analyzed T2 relaxation time values of ACI graft cartilage and the clinical results standardized after 6,12 , 24 , and 36 months.

In this study, all patients showed significant improvements in T2 relaxation time values for ACI graft cartilage within $6(P=.002)$ and 12 months $(P=.03)$. These significant improvements indicate a relevant change in the biochemical tissue parameters and provide a hint for graft maturation up to 1 year postoperatively. The $\mathrm{T} 2$ relaxation times improved from 41.6 milliseconds after 6 months to 30.9 milliseconds after 3 years. Nearly normal $\mathrm{T} 2$ relaxation time values for ACI graft cartilage were detected at 1 year postoperatively. This finding could represent the end of the maturing process of the ACI graft. After 1 year, the $\mathrm{T} 2$ relaxation time was not significantly different but still higher for ACI graft cartilage compared with the native surrounding cartilage. Completely harmonized $\mathrm{T} 2$ relaxation time values were found after 2 years postoperatively, which indicates completed maturation. At this point, no more differences in T2 relaxation times between graft cartilage and healthy cartilage could be found.

Welsch et $\mathrm{al}^{27,29}$ showed similar results after microfracture $(\mathrm{n}=10)$ and mb-ACI $(\mathrm{n}=10)$ in 20 patients. Within 1 to 2 years after surgery, graft cartilage had similar time values as healthy cartilage. No differences were found in localization in terms of MRI time values. However, the MRI follow-up was conducted after different time points and was not similar in both groups. A standardized MRI investigation was not performed in the postoperative course. A following study ${ }^{28}$ investigating the morphological parameters on MRI of 2 different ACI procedures
(Hyalograft C vs CaReS) showed comparable results after 24 months. However, differences were found in higher T2 values after ACI based on a collagen scaffold (CaReS).

In the present study, $\mathrm{T} 2$ relaxation mapping was performed in a standardized manner during the postoperative course of 3 years. The demonstrated results indicate that ACI grafts need at least 6 to 12 months for maturation. This assumption is supported by the results of a previous study. Watanabe et $\mathrm{al}^{26}$ found that ACI graft cartilage maturation was completed after 9 to 12 months. This is of major interest regarding the postoperative rehabilitation protocol. In a previous study, it was shown that early resumption of physical activities leads to inferior clinical outcomes after mb-ACI in the knee joint. ${ }^{17}$ Marlovits et $\mathrm{al}^{11}$ determined on the basis of the magnetic resonance observation of cartilage repair tissue (MOCART) scoring system that ACI graft maturation is completed after 24 months.

We used the IKDC subjective knee evaluation form and the VAS for subjective clinical evaluation. Both scores have often been used in previous studies that have dealt with the mb-ACI procedure. The clinical outcomes of the IKDC and VAS showed significantly higher results after 6,12 , 24 , and 36 months compared with the preoperative findings. However, the subjective IKDC score in this study population is low after 3 years, with a mean value of 57.3 and a high SD of 17.0. Nevertheless, we found a mean improvement from the preoperative subjective IKDC score of 27.6, which is comparable with scores in previous studies. $^{10,15,23}$ Twelve months postoperatively, the subjective IKDC scores were stable and significantly improved compared with the preoperative values. Regarding the question of influence of ACI graft maturation, disparities between the T2 relaxation time values and the detected results of the IKDC and VAS could not be found over the observation period. There was no significant correlation between clinical outcomes (IKDC) and T2 relaxation time values of ACI graft cartilage after 6, 12, 24, and 36 months. Also, we could not find a significant correlation between T2 relaxation time values and VAS scores of pain at rest $(P=$ $.3)$ and on movement $(P=.4)$ after 36 months and earlier examination periods $(P>.05)$ postoperatively.

One limitation of the study is the low number of cases. To obtain detailed information about ACI graft maturation and its clinical relevance, further prospective studies are necessary with a higher number of patients. Furthermore, it would be interesting to acquire more information about the cartilage structure and content of problematic ACI graft therapies with graft hypertrophy or postoperative pain.

Finally, the results of the current study demonstrate that ACI graft cartilage shows a significant improvement on T2 mapping over the observation period of 3 years, without a correlation with the clinical outcomes in IKDC and VAS scores. After 24 months postoperatively, no further T2 relaxation time differences between graft cartilage and healthy cartilage could be found. These findings support the theory that ACI graft maturation needs at least 12 months for regeneration and harmonization with the surrounding healthy cartilage. 


\section{CONCLUSION}

In this study, for the first time, we evaluated ACI graft maturation with $\mathrm{T} 2$ relaxation time values and its clinical relevance in IKDC scores within a postoperative course of 3 years. We found that ACI graft cartilage shows significant improvement in $\mathrm{T} 2$ relaxation time values over the whole observation period of 3 years. A correlation between T2 relaxation time values and clinical outcomes after 6,12 , 24 , and 36 months could not be found. These findings emphasize that ACI graft maturation needs at least 12 to 24 months before cartilage regeneration is completed.

\section{REFERENCES}

1. Alparslan L, Minas T, Winalski CS. Magnetic resonance imaging of autologous chondrocyte implantation. Semin Ultrasound CT MR. 2001;22(4):341-351.

2. Anders S, Schaumburger J, Schubert T, Grifka J, Behrens P. [Matrixassociated autologous chondrocyte transplantation (MACT): minimally invasive technique in the knee]. Oper Orthop Traumatol. 2008;20(3):208-219.

3. Behrens P, Bitter T, Kurz B, Russlies M. Matrix-associated autologous chondrocyte transplantation/implantation (MACT/MACl): 5-year follow-up. Knee. 2006;13(3):194-202.

4. Blackman AJ, Smith MV, Flanigan DC, Matava MJ, Wright RW, Brophy $\mathrm{RH}$. Correlation between magnetic resonance imaging and clinical outcomes after knee cartilage repair: author's response. Am J Sports Med. 2013;41(11):NP49-NP50.

5. Brittberg M, Lindahl A, Nilsson A, Ohlsson C, Isaksson O, Peterson L. Treatment of deep cartilage defects in the knee with autologous chondrocyte transplantation. N Engl J Med. 1994;331(14):889-895.

6. Dunn TC, Lu Y, Jin H, Ries MD, Majumdar S. T2 relaxation time of cartilage at MR imaging: comparison with severity of knee osteoarthritis. Radiology. 2004;232(2):592-598.

7. Eckstein F, Sittek H, Milz S, Putz R, Reiser M. The morphology of articular cartilage assessed by magnetic resonance imaging (MRI): reproducibility and anatomical correlation. Surg Radiol Anat. 1994;16(4):429-438.

8. Friedrich KM, Shepard T, de Oliveira VS, et al. T2 measurements of cartilage in osteoarthritis patients with meniscal tears. AJR Am J Roentgenol. 2009;193(5):W411-W415.

9. Higgins LD, Taylor MK, Park D, et al. Reliability and validity of the International Knee Documentation Committee (IKDC) Subjective Knee Form. Joint Bone Spine. 2007;74(6):594-599.

10. Kreuz PC, Muller S, von Keudell A, et al. Influence of sex on the outcome of autologous chondrocyte implantation in chondral defects of the knee. Am J Sports Med. 2013;41(7):1541-1548.

11. Marlovits S, Singer P, Zeller P, Mandl I, Haller J, Trattnig S. Magnetic resonance observation of cartilage repair tissue (MOCART) for the evaluation of autologous chondrocyte transplantation: determination of interobserver variability and correlation to clinical outcome after 2 years. Eur J Radiol. 2006;57(1):16-23.

12. Mendlik T, Faber SC, Weber J, et al. T2 quantitation of human articular cartilage in a clinical setting at $1.5 \mathrm{~T}$ : implementation and testing of four multiecho pulse sequence designs for validity. Invest Radiol. 2004;39(5):288-299.

13. Mosher TJ, Dardzinski BJ. Cartilage MRI T2 relaxation time mapping: overview and applications. Semin Musculoskelet Radiol. 2004;8(4):355-368.

14. Niemeyer $\mathrm{P}$, Andereya $\mathrm{S}$, Angele $\mathrm{P}$, et al. Autologous chondrocyte implantation $(\mathrm{ACl})$ for cartilage defects of the knee: a guideline by the working group "Tissue Regeneration" of the German Society of
Orthopaedic Surgery and Traumatology (DGOU). Z Orthop Unfall. 2013;151(1):38-47.

15. Niemeyer P, Pestka JM, Salzmann GM, Sudkamp NP, Schmal H. Influence of cell quality on clinical outcome after autologous chondrocyte implantation. Am J Sports Med. 2012;40(3):556-561.

16. Niemeyer $\mathrm{P}$, Porichis $\mathrm{S}$, Steinwachs $\mathrm{M}$, et al. Long-term outcomes after first-generation autologous chondrocyte implantation for cartilage defects of the knee. Am J Sports Med. 2014;42(1):150-157.

17. Niethammer TR, Muller PE, Safi E, et al. Early resumption of physical activities leads to inferior clinical outcomes after matrix-based autologous chondrocyte implantation in the knee. Knee Surg Sports Traumatol Arthrosc. 2014;22(6):1345-1352.

18. Niethammer TR, Pietschmann MF, Horng A, et al. Graft hypertrophy of matrix-based autologous chondrocyte implantation: a two-year follow-up study of NOVOCART 3D implantation in the knee. Knee Surg Sports Traumatol Arthrosc. 2014;22(6):1329-1336.

19. Nissi MJ, Toyras J, Laasanen MS, et al. Proteoglycan and collagen sensitive MRI evaluation of normal and degenerated articular cartilage. J Orthop Res. 2004;22(3):557-564.

20. Pestka JM, Bode G, Salzmann G, et al. Clinical outcomes after cellseeded autologous chondrocyte implantation of the knee: when can success or failure be predicted? Am J Sports Med. 2014;42(1):208-215.

21. Pietschmann MF, Niethammer TR, Horng A, et al. The incidence and clinical relevance of graft hypertrophy after matrix-based autologous chondrocyte implantation. Am J Sports Med. 2012;40(1):68-74.

22. Risberg MA, Holm I, Steen H, Beynnon BD. Sensitivity to changes over time for the IKDC form, the Lysholm score, and the Cincinnati knee score: a prospective study of $120 \mathrm{ACL}$ reconstructed patients with a 2-year follow-up. Knee Surg Sports Traumatol Arthrosc. 1999;7(3):152-159.

23. Schneider U, Rackwitz L, Andereya S, et al. A prospective multicenter study on the outcome of type I collagen hydrogel-based autologous chondrocyte implantation (CaReS) for the repair of articular cartilage defects in the knee. Am J Sports Med. 2011;39(12):2558-2565.

24. Trattnig S, Ba-Ssalamah A, Pinker K, Plank C, Vecsei V, Marlovits S. Matrix-based autologous chondrocyte implantation for cartilage repair: noninvasive monitoring by high-resolution magnetic resonance imaging. Magn Reson Imaging. 2005;23(7):779-787.

25. Trattnig S, Pinker K, Krestan C, Plank C, Millington S, Marlovits S. Matrix-based autologous chondrocyte implantation for cartilage repair with HyalograftC: two-year follow-up by magnetic resonance imaging. Eur J Radiol. 2006;57(1):9-15.

26. Watanabe A, Wada $Y$, Obata T, et al. Time course evaluation of reparative cartilage with MR imaging after autologous chondrocyte implantation. Cell Transplant. 2005;14(9):695-700.

27. Welsch GH, Mamisch TC, Domayer SE, et al. Cartilage T2 assessment at 3-T MR imaging: in vivo differentiation of normal hyaline cartilage from reparative tissue after two cartilage repair procedures. Initial experience. Radiology. 2008;247(1):154-161.

28. Welsch GH, Mamisch TC, Zak L, et al. Evaluation of cartilage repair tissue after matrix-associated autologous chondrocyte transplantation using a hyaluronic-based or a collagen-based scaffold with morphological MOCART scoring and biochemical T2 mapping: preliminary results. Am J Sports Med. 2010;38(5):934-942.

29. Welsch GH, Trattnig S, Domayer S, Marlovits S, White LM, Mamisch TC. Multimodal approach in the use of clinical scoring, morphological MRI and biochemical T2-mapping and diffusion-weighted imaging in their ability to assess differences between cartilage repair tissue after microfracture therapy and matrix-associated autologous chondrocyte transplantation: a pilot study. Osteoarthritis Cartilage. 2009;17(9):1219-1227.

30. Wondrasch B, Zak L, Welsch GH, Marlovits S. Effect of accelerated weightbearing after matrix-associated autologous chondrocyte implantation on the femoral condyle on radiographic and clinical outcome after 2 years: a prospective, randomized controlled pilot study. Am J Sports Med. 2009;37(Suppl 1):88S-96S. 